\title{
Comportamento térmico de compósitos PS-HDL (Mg-Al) modificados com DBS e SDS
}

\section{Thermal behavior of composites of PS-LDH (Mg-Al) modified with SDB and SDS}

\author{
Lucas Dominguini ${ }^{1 *}$, Renata Guidi da Rosa ${ }^{2}$, Karina Martinello², Juliana Pavei Pizzolo ${ }^{2}$ \\ Márcio Antônio Fiori ${ }^{3}$ \\ 'Departamento de Química, Instituto Federal de Educação, Ciência e Tecnologia de \\ Santa Catarina - IFSC, Criciúma, SC, Brasil \\ ${ }^{2}$ Departamento de Engenharia Química, Universidade do Extremo Sul Catarinense - UNESC, \\ Criciúma, SC, Brasil \\ ${ }^{3}$ Programa de Pós-Graduação em Ciências Ambientais, Universidade Comunitária da Região de \\ Chapecó - UNOCHAPECÓ, Chapecó, SC, Brasil \\ *lucas.dominguini@ifsc.edu.br
}

\begin{abstract}
Resumo
Hoje em dia é crescente a quantidade de estudos envolvendo a utilização de cargas ou aditivos nos materiais poliméricos, em especial no poliestireno, a fim de atuar como retardante de chama. Partindo desse princípio, este trabalho compara o comportamento térmico dos compósitos de poliestireno/hidróxido duplo lamelar magnésio-alumínio (PS-HDL [Mg-Al]) em relação ao poliestireno puro. Os compostos foram preparados com HDL, quimicamente modificado com dodecil sulfato de sódio (SDS-Na) ou com dodecilbenzeno sulfonato de sódio (DBS-Na). A síntese desses aditivos (HDL-SDS e HDL-DBS) foi feita pelo método de coprecipitação e inseridos no PS via solubilização em clorofórmio. Os compostos foram caracterizados por difratometria de raios-X e espectroscopia de infravermelho por transformada de Fourier, onde o primeiro comprovou a intercalação do DBS/SDS com o HDL e o segundo confirmou a presença dos grupos característicos no HDL. Através da análise termogravimétrica, avaliaram-se as propriedades térmicas do compósito. Os resultados mostraram que o PS-HDL modificado comporta-se melhor que o PS puro, pois apresenta maior estabilidade térmica. Comparando-os em uma perda de massa de 50\%, o PS-HDL-SDS apresentou resultado superior ao PS puro, com um ganho de aproximadamente $44{ }^{\circ} \mathrm{C}$ na temperatura de decomposição térmica.
\end{abstract}

Palavras-chave: comportamento térmico, poliestireno, hidróxido duplo lamelar, modificação química.

\begin{abstract}
Nowadays there is an increasing number of studies involving the use of fillers or additives for polymeric materials, in particular polystyrene, as flame retardants. Starting from this principle, this paper compares the thermal performance of composites of polystyrene/double layered magnesium-aluminium hydroxide (PS-LDH [Mg-Al]) compared to pure polystyrene. Compounds were prepared with $\mathrm{LDH}$, chemically modified with sodium dodecyl sulphate (SDS-Na) or sodium dodecylbenzene sulfonate (SDB-Na). The synthesis of these additives (SDS-LDH and SDB-LDH) was carried out by the coprecipitation method and inserted via the PS dissolution in chloroform. The compounds were characterized by X-ray diffraction and Fourier transform infrared spectroscopy, where the first method proved the intercalation of the $\mathrm{SBD} / \mathrm{SDS}$ with LDH and the second confirms the presence of the characteristic groups in LDH. Thermal properties of the composite were evaluated by thermo gravimetric analysis. The results showed that the modified PS-LDH behaves better than pure PS, it has higher thermal stability. Comparing them in a mass loss of $50 \%$, the PS-LDH-SDS showed better results compared to pure PS, with a gain of approximately $44{ }^{\circ} \mathrm{C}$ in the thermal decomposition temperature.
\end{abstract}

Keywords: thermal behavior, polystyrene, lamellar double hydroxide, chemical modification.

\section{Introdução}

O poliestireno (PS), fórmula molecular $\left(\mathrm{C}_{8} \mathrm{H}_{8}\right)_{x}$, é um termoplástico cuja característica reside na sua alta flexibilidade ou fácil maleabilidade sob ação do calor. Suas propriedades

e características permitem uma ampla gama de aplicações em automóvel, construção, embalagem, eletroeletrônica entre outras ${ }^{[1,2]}$. 
O PS exposto a uma fonte de calor sob temperatura elevada sofre degradação térmica com a geração de gases combustíveis inflamáveis. A combustão destes gases gera chamas e elevação da temperatura, no qual atinge valores entre $350{ }^{\circ} \mathrm{C}$ a $500{ }^{\circ} \mathrm{C}$, podendo chegar a temperatura de $900{ }^{\circ} \mathrm{C}$ a $1000{ }^{\circ} \mathrm{C}$. Após o consumo do material orgânico pela combustão, ocorre o decaimento e extinção da chama ${ }^{[3]}$.

A combustão do poliestireno produz toxinas que apresentam riscos à saúde dos seres vivos. Assim, é crescente a quantidade de estudos envolvendo a utilização de cargas ou aditivos para os materiais poliméricos, em especial no poliestireno, a fim de aumentar sua estabilidade térmica ${ }^{[4]}$.

Durante a degradação térmica de um polímero é gerado subprodutos, como radicais de elevada reatividade capazes de interagir com o oxigênio presente na atmosfera. A liberação dos gases na atmosfera promove reações de desidrogenação, originando radicais de elevada reatividade do tipo $\mathrm{H} \bullet, \mathrm{OH} \bullet$ e $\mathrm{O} \cdot$. Estes radicais impulsionam a formação da chama a fim de obter o equilíbrio termodinâmico. Dessa forma, a temperatura do início da decomposição é mais baixa do que a temperatura de ignição. Os ciclos de combustão são divididos em duas fases, a fase condensada, onde ocorrem os efeitos que são responsáveis pelo início do ciclo de queima, e a fase gasosa, com os gases provenientes da combustão do polímero ${ }^{[5]}$.

Um agente retardante de chama inorgânico, que tem a função de prolongar o período de ignição ou até mesmo a extinção da chama, atua na fase condensada. As reações entre os agentes retardantes de chamas e os radicais livres resultam na liberação de gases não combustíveis do tipo dióxido de carbono $\left(\mathrm{CO}_{2}\right)$ e água $\left(\mathrm{H}_{2} \mathrm{O}\right)$. Estas reações reduzem a concentração de oxigênio $\left(\mathrm{O}_{2}\right)$ na superfície do material polimérico e inibem a reação de combustão pela supressão de oxigênio. Concomitante com as reações de supressão de oxigênio os resíduos da combustão dos agentes retardantes contribuem para diminuir a superfície de contato entre combustível e comburente, formando revestimentos nas moléculas poliméricas e o isolamento dos sítios de combustão ${ }^{[6]}$.

Uma das classes de agentes retardantes de chamas empregados em polímeros são os compostos de hidróxidos duplos lamelares denominados por HDL. Os HDL's são materiais com estrutura semelhante à da brucita $\left(\mathrm{Mg}(\mathrm{OH})_{2}\right)$, resultado da substituição isomórfica de uma parcela dos cátions $\mathrm{Mg}^{2+}$ por um cátion trivalente $\mathrm{M}^{3+}$, que pode ser o $\mathrm{Al}^{3+[7]}$. Essas estruturas apresentam formulação genérica do tipo $\left[\mathrm{M}^{2+}{ }_{1-x} \mathrm{M}^{3+}{ }_{\mathrm{x}}(\mathrm{OH})_{2}\right]\left[\mathrm{A}^{\mathrm{m}-}{ }_{\mathrm{x} / \mathrm{m}} \cdot \mathrm{nH}_{2} \mathrm{O}\right]$, onde $\mathrm{M}^{2+}$ representa um cátion bivalente, $\mathrm{M}^{3+}$ representa um cátion trivalente,
$\mathrm{A}^{\mathrm{m}-}$ representa um ânion e $n$ representa o número de moléculas de água ${ }^{[8,9]}$.

As características que tornam os HDL's agentes promissores na obtenção de compósitos poliméricos resistentes à queima são suas estruturas cristalinas lamelares e sua habilidade de realizar troca iônica com as espécies aniônicas contidas nas suas lamelas. Essas características dos HDL's facilitam as interações de sua estrutura inorgânica com o polímero e assim, quando submetidos ao aquecimento sofrem decomposição térmica. Tal fenômeno ocorre em três etapas: (i) liberação da água de hidratação em aproximadamente $200{ }^{\circ} \mathrm{C}$, (ii) decomposição das hidroxilas na faixa de $200{ }^{\circ} \mathrm{C}$ a $450{ }^{\circ} \mathrm{C}$ e (iii) calcinação e formação de óxidos duplos entre a faixa de $450{ }^{\circ} \mathrm{C}$ a $600{ }^{\circ} \mathrm{C}^{[4,10]}$.

$\mathrm{Na}$ condição de calcinação os HDL's sofrem uma reação endotérmica, decompondo-se em vapor de água e óxidos metálicos. Nesse processo, três fatores atuam em sinergismo para retardar a combustão do material polimérico: (i) absorção de calor pela reação endotérmica, (ii) liberação de vapor de água que dilui a concentração de oxigênio do ar e (iii) formação de uma camada de óxidos que atuam como barreira térmica e química do calor e do oxigênio gasoso com a matriz polimérica, respectivamente. Este protetor térmico formado pode proporcionar um tempo de ignição maior, muitas vezes suficientes para permitir o resgate de pessoas expostas ao risco de combustão ou ainda diminuir a velocidade de propagação das chamas ${ }^{[1,12]}$.

Esses compostos inorgânicos apresentam baixo acoplamento com a matriz polimérica porque são altamente hidrofílicos, enquanto a matriz polimérica é hidrofóbica. Para melhorar esse acoplamento da matriz orgânica com o aditivo inorgânico, são feitas substituições de íon hidroxila $\left(\mathrm{OH}^{-}\right)$por um íon com terminações hidrofóbicas, para atuarem como agentes surfactantes. Duas possibilidades de compostos com tais características podem ser utilizados que são o dodecilsulfato de sódio (SDS-Na) e o dodecilbenzenosulfonato de sódio (DBS-Na). Sua longa calda orgânica apolar e sua extremidade iônica facilitam a interação entre as fases orgânicas (polímero) e inorgânicas (HDL), diminuindo a tensão superficial e melhorando a compatibilidade entre o agente e a matriz polimérica. Na Figura 1 são mostradas as estruturas do SDS-Na e DBS-Na ${ }^{[13]}$.

Nesse caso, íons hidroxilas presentes na estrutura do HDL podem ser substituídos por íons SDS ou DBS, por troca iônica. Com isso, modifica-se a estrutura do HDL, facilitando o seu acoplamento com a matriz polimérica. Vários fatores influenciam a estrutura intercalada do HDL na matriz polimérica e, por consequência, as características do processo de combustão do polímero. Como exemplo o

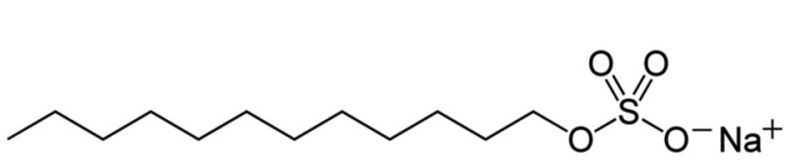<smiles>CCCCc1ccc(S(=O)(=O)O[Na])cc1</smiles>

(a)

(b)

Figura 1. (a) Estrutura química do SDS-Na, (b) Estrutura química do DBS. 
tempo de refluxo, a concentração de HDL no solvente, o tipo de solvente e método de preparação ${ }^{[14-18]}$.

São encontrados relatos sobre incorporação de HDL em poliestireno de diferentes formas: (i) durante a polimerização do monômero de estireno, via emulsão ${ }^{[14,15]}$, (ii) na síntese in situ com a mistura dos monômeros e do HDL ${ }^{[16]}$, (iii) na preparação via intercalação de poliestireno em solução de xileno para formar PS-HDL ${ }^{[17]} \mathrm{e}$ (iv) incorporação física no polímero via os processos de extrusão ou fusão ${ }^{[18]}$. Porém, não há relatos de trabalho envolvendo a inserção do HDL em PS via solubilização em clorofórmio $\left(\mathrm{CHCl}_{3}\right)$.

Neste trabalho a incorporação do HDL em PS foi realizada via solubilização em clorofórmio e mistura mecânica dos componentes. Os HDL's utilizados foram do tipo $\mathrm{Mg}$-Al, com e sem ânions de SDS e DBS intercalados nas suas lamelas. Assim, foi avaliada a estabilidade térmica dos compósitos do tipo PS-HDL, PS-HDL-SDS, PS-HDL-DBS comparados com do PS puro.

\section{Materiais e Métodos}

\subsection{Sintese do HDL-SDS e HDL-DBS}

Para a preparação dos HDL's quimicamente modificados, utilizou-se como reagentes o dodecilsulfato de sódio $\left[\mathrm{C}_{12} \mathrm{H}_{25} \mathrm{NaO}_{4} \mathrm{~S}\right]$ (Sigma, 99\% de pureza), dodecilbenzeno sulfonato de sódio $\left[\mathrm{C}_{18} \mathrm{H}_{29} \mathrm{NaO}_{3} \mathrm{~S}\right]$ (Sigma, $99 \%$ de pureza), nitrato de magnésio hexa-hidratado $\left[\mathrm{Mg}\left(\mathrm{NO}_{3}\right)_{2} \cdot 6 \mathrm{H}_{2} \mathrm{O}\right]$ (Vetec, 98\% de pureza), nitrato de alumínio nona-hidratado [Al $\left.\left(\mathrm{NO}_{3}\right)_{3} .9 \mathrm{H}_{2} \mathrm{O}\right]$ (Vetec, $98 \%$ de pureza) e hidróxido de sódio $(\mathrm{NaOH})$ (Vetec, $99,6 \%$ de pureza).

Os HDL's foram sintetizados pelo método de coprecipitação. Em um reator de vidro contendo uma solução aquosa de SDS-Na, 0,033 mol/L, foi adicionado lentamente (gota a gota) uma solução de nitrato de magnésio, $0,066 \mathrm{~mol} / \mathrm{L}$, de nitrato de alumínio, $0,033 \mathrm{~mol} / \mathrm{L}$ e, simultaneamente, a solução básica de $\mathrm{NaOH}, 1,2 \mathrm{~mol} / \mathrm{L}$. O pH foi monitorado e mantido com valor igual a 10 no meio reacional.

Os reagentes permaneceram em agitação constante durante sete horas para a precipitação e crescimento dos cristais do HDL Mg-Al modificado com SDS-Na. A suspensão formada foi lavada com água deionizada repetidamente até remoção dos reagentes em excesso, evitando o surgimento contaminantes no momento da caracterização do material. O sólido obtido foi seco em estufa com temperatura controlada de $60^{\circ} \mathrm{C}$. Após a secagem o produto foi macerado.

A obtenção do HDL-DBS foi realizada de forma semelhante, com a substituição do SDS-Na pelo DBS-Na.

\subsection{Caracterização do HDL-SDS e HDL-DBS}

Para caracterização dos HDL's foi empregada a técnica de difratometria de raios-X (DRX) (Shimadzu, XRD-6000, alvo de cobre), com diferença de potencial de $25 \mathrm{kV}$ e corrente de $25 \mathrm{~mA}$. Os padrões foram obtidos com velocidade de passo igual a $2,0^{\circ} \cdot \mathrm{s}^{-1}$, com varredura de 2 a $80^{\circ}(2 \theta)$. A intercalação foi avaliada via DRX de acordo com o espaçamento basal do HDL Mg-Al padrão e dos HDL's quimicamente modificados com SDS e DBS.

Para comprovar a presença dos grupos característicos dos compostos intercalados no HDL Mg-Al foi empregada a técnica de espectroscopia de infravermelho com transformada de Fourier (FTIR) (Shimadzu, IR Prestige21), com a avaliação de número de onda na região de 4.000 a $500 \mathrm{~cm}^{-1}$.

\subsection{Produção dos compósitos PS-HDL}

Para preparação dos compósitos utilizou-se PS (Videolar, GPPS 535) com índice de fluidez de 5,0 g/10 min, à $200{ }^{\circ} \mathrm{C}$, massa específica de $1,04 \mathrm{~g} \cdot \mathrm{cm}^{-1}$, com temperatura de amolecimento de $106{ }^{\circ} \mathrm{C}$, conforme ficha técnica disponibilizada pelo fabricante.

Na preparação dos compósitos PS-HDL, PS-HDL-SDS e PS-HDL-DBS utilizou-se a técnica de solubilização, tendo como solvente o clorofórmio. O PS foi solubilizado em clorofórmio (Fmaia, 99,8\% de pureza), na proporção mássica de 1:10, com auxílio de um agitador mecânico, até obtenção de uma solução homogênea. A incorporação dos HDL's na matriz polimérica foi efetuada após solubilização total do PS por mistura mecânica dos constituintes, com 30\% em massa de HDL. Após a obtenção da mistura homogênea, o solvente foi extraído por evaporação em estufa em $60{ }^{\circ} \mathrm{C}$. Também foram preparados corpos de prova de PS puro para fins de comparação.

\subsection{Caracterização do compósito PS-HDL quimicamente modificado}

A aditivação do PS com os HDL's quimicamente modificados foi confirmada através de análises de espectroscopia de infravermelho com transformada de Fourier (FTIR) (Shimadzu, IR Prestige-21) com a avaliação de número de onda na região de 4.000 a $500 \mathrm{~cm}^{-1}$. Nos espectros obtidos foram comparadas as bandas dos HDL's modificados, do PS e dos compósitos PS-HDL, PS-HDL-SDS e PS-HDL-DBS.

Para avaliar a estabilidade térmica dos compósitos em relação ao PS puro foi empregada a técnica de análise termogravimétrica (TGA) (Shimadzu TGA-50). A fim de caracterizar as variações mássicas das amostras, através do aumento controlado da temperatura, os ensaios foram realizados partindo da temperatura ambiente até $900{ }^{\circ} \mathrm{C}$, com taxa de aquecimento de $10^{\circ} \mathrm{C} \cdot \mathrm{min}^{-1} \mathrm{e}$ em atmosfera inerte de nitrogênio de alta pureza de $50 \mathrm{~mL} \cdot \mathrm{min}^{-1}$.

\section{Resultados e Discussão}

\subsection{Caracterização do HDL-SDS e HDL-DBS}

As Figuras 2 e 3 mostram os difratogramas obtidos para o HDL puro, HDL-DBS e HDL-SDS, respectivamente. Ambos os espectros apresentam características que indicam modificações significativas na estrutura cristalina do HDL após o tratamento com DBS e SDS.

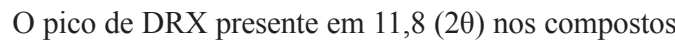
de HDL é relativo ao espaçamento basal da sua estrutura. A intercalação dos íons SDS e DBS na região interlamelar da estrutura do HDL reflete no deslocamento deste pico para valores menores de ângulos no espectro, que implica na confirmação do aumento do espaçamento basal da estrutura do HDL. O aumento do espaçamento interlamelar pode ser comprovado através do deslocamento do pico característico d001 para ângulos menores ${ }^{[19]}$. 


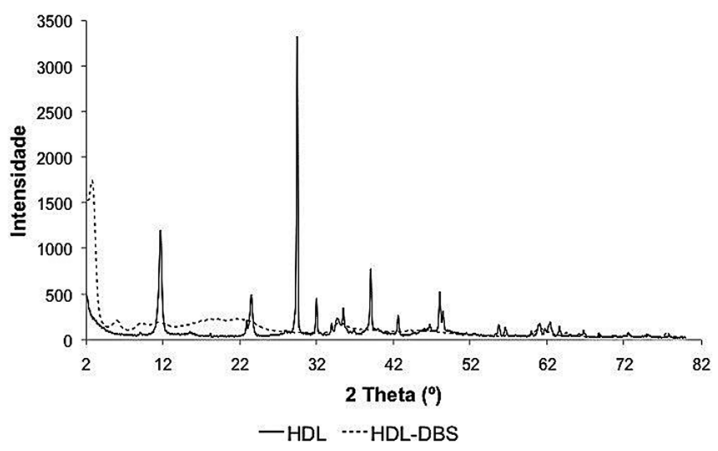

Figura 2. Difratograma de raios-X para o HDL e HDL-DBS.

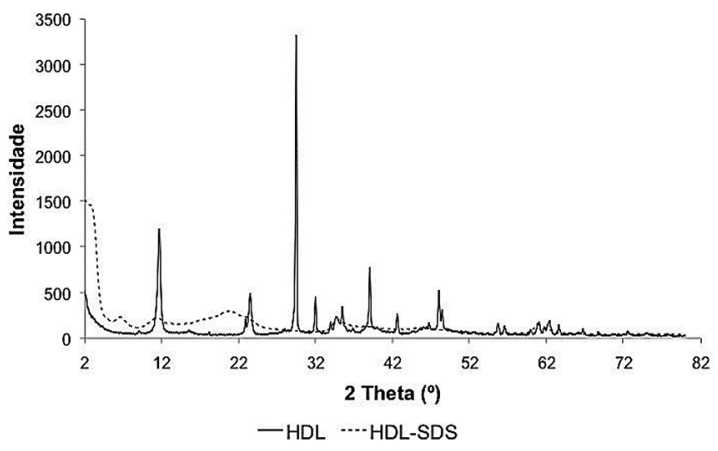

Figura 3. Difratograma de raios-X para o HDL e HDL-SDS.

A Figura 4 evidencia o deslocamento do pico d001 com a presença de DBS e SDS na estrutura do HDL. Para o HDL-DBS, o pico desloca-se para $2 \theta=2,82^{\circ} \mathrm{e}$, para o HDL-SDS, o pico desloca-se para $2 \theta=3,02$. A estrutura do DBS possui anéis aromáticos e proporcionam moléculas com raio moleculares maiores. Consequentemente, a sua intercalação promove espaçamentos interlamelares na estrutura dos HDL's maiores e menores ângulos para o pico característico no difratograma.

Os resultados de difração de raios-X indicam um valor de espaçamento lamelar de 0,762 nm para o HDL puro. Após o processo de intercalação os espaçamentos interlamelares do HDL são de 3,13 nm para HDL-DBS e de 2,92 nm para HDL-SDS.

\subsection{Ensaio de FTIR}

As Figuras 5 e 6 mostram os espectros de FTIR obtidos para os compostos de HDL puro, HDL-SDS e HDL-DBS. É possível observar a banda intensa e larga na região de $3500 \mathrm{~cm}^{-1}$, característica do grupo hidroxila, presente no HDL. As bandas de estiramento da ligação carbono-hidrogênio (C-H), na região de $2800 \mathrm{~cm}^{-1}$, estão presentes no cátion SDS e nos compostos de HDL, conforme mostra Figura 5. A presença da banda na região de $1240 \mathrm{~cm}^{-1}$ e de uma banda menos intensa em 800 a $820 \mathrm{~cm}^{-1}$ é um indicativo de que ocorreu a sulfonação da estrutura do HDL ${ }^{[13]}$. Os espectros de FTIR para o HDL-DBS evidenciam que houve intercalação do cátion DBS pelo surgimento de bandas na região de $1000 \mathrm{~cm}^{-1}$, que corresponde ao estiramento $\mathrm{S}=\mathrm{O}$ do $\mathrm{DBS}^{[13]}$.

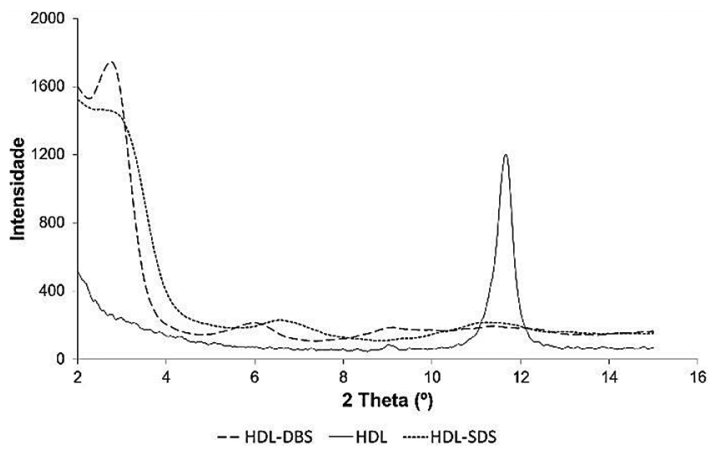

Figura 4. Difratograma de raios-X para baixos ângulos para HDL, HDL-DBS e HDL-SDS.

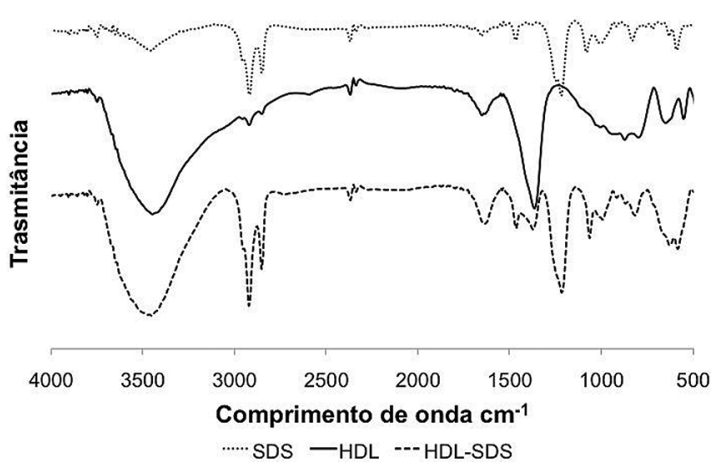

Figura 5. Espectro de infravermelho (FTIR) do SDS, HDL e HDL-SDS.

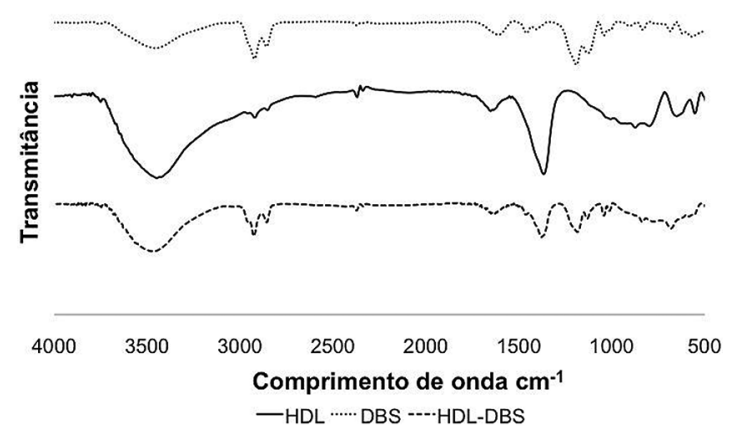

Figura 6. Espectro de infravermelho (FTIR) do DBS, HDL e HDL-DBS.

Os resultados das análises de FTIR mostram a formação de um composto híbrido, contendo na estrutura de HDL as moléculas de SDS e DBS. Os espectros resultantes do HDL-DBS e do HDL-SDS apresentam todas as bandas relativas aos modos de vibração da estrutura do HDL puro e das moléculas puras de DBS e de SDS. Esta característica comprova que no processo de síntese ocorreram apenas interações físicas, resultando apenas na intercalação das moléculas de DBS e de SDS nos espaçamentos basais da estrutura do HDL. Estes resultados corroboram com os resultados de difração de raios-X, que indica a intercalação das moléculas na estrutura cristalina do HDL. 


\subsection{Análise Termogravimétrica (TGA)}

A Figura 7 ilustra os termogramas para o poliestireno puro (PS), para o compósito PS-HDL e para os compósitos PS-HDL-SDS e PS-HDL-DBS. Os resultados indicam a decomposição térmica do poliestireno puro a partir de $380^{\circ} \mathrm{C}$, sendo finalizada $\mathrm{em} 430^{\circ} \mathrm{C}$. A massa residual resultante do processo de queima do PS é praticamente nula.

A incorporação de HDL puro no PS, compósito PS-HDL, proporciona o aumento da temperatura de partida da degradação térmica do PS e o aumento da massa residual após o processo de combustão para aproximadamente $9 \%$ em massa. $\mathrm{O}$ termograma mostra também a liberação de água adsorvida na estrutura do HDL.

A incorporação de HDL-SDS e de HDL-DBS no poliestireno resulta em um compósito com maior temperatura de degradação térmica e maior massa residual após o processo de queima. A primeira etapa da perda de massa observada para o PS-HDL-SDS é na região de 200 a $250^{\circ} \mathrm{C}$, devido à perda da água fisicamente adsorvida nas camadas

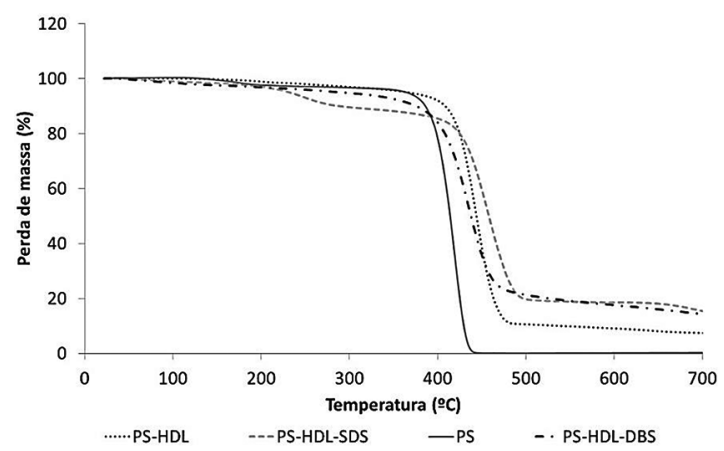

Figura 7. Termogramas para PS puro e compósitos de PS-HDL, PS-HDL-DBS e PS-HDL-SDS. intercaladas. A segunda etapa de perda de massa é na região de 300 a $450{ }^{\circ} \mathrm{C}$ decorrente da degradação térmica do PS.

A taxa de degradação é muito mais lenta para os compostos contendo HDL do que o PS puro. Este efeito está associado com a dificuldade proporcionada pelas camadas de HDL para a difusão de oxigênio e os produtos voláteis no compósito, tendo como consequência a redução da cinética do processo de combustão ${ }^{[13]}$.

A incorporação de HDL-SDS e HDL-DBS no compósito com matriz polimérica de poliestireno resultam em benefícios em relação a temperatura de início de degradação térmica, que reflete no aumento da estabilidade térmica e na redução da taxa de combustão, no qual resulta em um efeito de retardância de chamas no compósito. Ainda, resulta em maior massa residual de 19\% em massa. Este efeito proporciona maior proteção das moléculas poliméricas durante o processo de combustão e resulta em maiores dificuldades de propagação de chamas no compósito.

Considerando a perda de $50 \%$ de massa no processo de degradação térmica, as temperaturas de decomposição térmica para o PS puro, PS-HDL, PS-HDL-SDS e PS-HDL-DBS são de $414{ }^{\circ} \mathrm{C}, 444{ }^{\circ} \mathrm{C}, 458{ }^{\circ} \mathrm{C}$ e $438{ }^{\circ} \mathrm{C}$, respectivamente. Esta comparação mostra que a temperatura de degradação térmica do PS é aumentada pela incorporação dos compostos de HDL, resultando em maior estabilidade térmica em comparação ao PS puro, ótimo indicador para aplicações futuras como agentes retardantes de chamas para o poliestireno $0^{[13]}$.

A Tabela 1 mostra os resultados obtidos com trabalhos similares envolvendo a adição de HDL em PS.

Os resultados evidenciam que a adição de um agente de intercalação de fase aumenta a massa residual do material polimérico. Provavelmente, a presença da cadeia orgânica dos ânions SDS e DBS melhor interagem com o polímero, o que aumenta sua estabilidade térmica.

Tabela 1. Relação entre a estrutura química do HDL, o método de incorporação na matriz polimérica, concentração mássica e a temperatura com $50 \%$ de perda de massa, em poliestireno.

\begin{tabular}{|c|c|c|c|c|c|c|}
\hline $\begin{array}{c}\text { Estrutura do } \\
\text { HDL }\end{array}$ & $\begin{array}{c}\text { Modificação } \\
\text { Química }\end{array}$ & $\begin{array}{c}\text { Método de } \\
\text { incorporação }\end{array}$ & $\begin{array}{l}\text { Concentração } \\
\text { Mássica (\%) }\end{array}$ & $\begin{array}{c}\text { Temperatura } \\
\text { com } 50 \% \text { de } \\
\text { perda de massa } \\
\left({ }^{\circ} \mathrm{C}\right)\end{array}$ & $\begin{array}{c}\text { Massa residual a } \\
600{ }^{\circ} \mathrm{C}(\%)\end{array}$ & Referência \\
\hline \multirow{3}{*}{ HDL-MgAl } & - & \multirow{3}{*}{$\begin{array}{l}\text { Solubilização em } \\
\text { Clorofórmio }\end{array}$} & 30 & 444 & 9 & \multirow{3}{*}{-} \\
\hline & SDS & & 30 & 458 & 19 & \\
\hline & DBS & & 30 & 438 & 19 & \\
\hline \multirow[b]{2}{*}{ HDL-ZnAl } & \multirow[b]{2}{*}{-} & \multirow[b]{2}{*}{ Emulsão } & 5 & 425 & 3 & \multirow{2}{*}{ Ding e $\mathrm{Qu}^{[14]}$} \\
\hline & & & 30 & 355 & 17 & \\
\hline HDL-ZnAl & SDS & In situ & 5 & 435 & 5 & $\operatorname{Botan}^{[13]}$ \\
\hline HDL-ZnAl & SDS & $\begin{array}{c}\text { Solubilização em } \\
\text { xileno }\end{array}$ & 20 & 367 & 9 & Qiu et al. ${ }^{[17]}$ \\
\hline HDL-MgAl & $\begin{array}{c}\text { Ácido } \\
\text { 10-undecenóico }\end{array}$ & Extrusão ou fusão & 10 & 431 & 2,5 & Nyambo et al. ${ }^{[18]}$ \\
\hline HDL-ZnAl & $\begin{array}{l}\text { 3-sulfopropil } \\
\text { metacrilato de } \\
\text { potássio }\end{array}$ & $\begin{array}{l}\text { Polimerização em } \\
\text { massa utilizando } \\
\text { peróxido de } \\
\text { benzoíla }\end{array}$ & 15 & 390 & 22 & Leroux et al. ${ }^{[20]}$ \\
\hline HDL-Ca-Al & Benzoato & In situ & 10 & 434 & 9,2 & Matusinovic et al. ${ }^{[9]}$ \\
\hline HDL-MgAl & $\begin{array}{l}\text { Bis(2-etil-hexil) } \\
\text { fosfato, DBS }\end{array}$ & $\begin{array}{c}\text { Fusão e } \\
\text { polimerização em } \\
\text { massa }\end{array}$ & 10 & 435 & 4 & Wang et al. ${ }^{[21]}$ \\
\hline
\end{tabular}


A técnica de inserção do HDL quimicamente modificado, por solubilização em clorofórmio, conferiu uma maior temperatura de perda de massa a $50 \%$, bem como maior resíduo mássico, quando comparado aos demais trabalhos.

\section{Conclusão}

Os resultados das sínteses de compósitos de PS-HDL e PS-HDL modificados com SDS e DBS mostraram-se satisfatórias para os ensaios de DRX, onde houve a intercalação dos aditivos no HDL, e comprovado através do FTIR a presença dos grupos funcionais na estrutura do HDL.

Os compósitos com HDL ou HDL modificados apresentaram maior estabilidade térmica que a do PS puro. Dentre os compósitos modificados, destaca-se o compósito PS-HDL-SDS onde foi obtido um aumento de $44{ }^{\circ} \mathrm{C}$ na temperatura de decomposição a $50 \%$ de perda de massa como ponto de comparação. Dessa forma, a técnica de inserção do HDL quimicamente modificado na matriz orgânica apresentou resultados satisfatórios em relação a outros métodos, principalmente por não necessitar de aquecimento para a sua realização.

\section{Agradecimentos}

Ao Programa Institucional de Bolsas de Iniciação Científica (PIBIC) e ao CNPq pelo financiamento da pesquisa.

\section{Referências}

1. Canevarolo, S. V., Jr. (2006). Ciência dos polímeros: um texto básico para tecnólogos e engenheiros. São Paulo: Artliber.

2. Xia, Y., Mao, Z., Jin, F., Guan, Y., \& Zheng, A. (2014). Synthesis of 1-hydroxy ethylidene-1,1-diphosphonic ammonium and the promise of this ammonium salt as an intumescent flame retardant in polystyrene. Polymer Degradation \& Stability, 102(4), 186194. http://dx.doi.org/10.1016/j.polymdegradstab.2014.01.011.

3. Kuljanin-Jakovljevic, J., Marinovic'-Cincovic, M., Stojanovic', Z., Krkljes, A., Abazovic, N. D., \& Comor, M. I. (2009). Thermal degradation kinetics of polystyrene/cadmium sulfide composites. Polymer Degradation \& Stability, 94(6), 891-897. http://dx.doi.org/10.1016/j.polymdegradstab.2009.03.004.

4. Becker, C. M., Gabbardo, A. D., Wypych, F., Amico S. C. (2009). Estudo das características mecânicas e de imflamabilidade de compósitos Epóxi/HDL. In Anais do $10^{\circ}$ congresso Brasileiro de Polímeros (pp. 1-10). Foz do Iguaçú: ABPol.

5. Gallo, J. B., \& Agnelli, A. M. (1998). Aspecto do comportamento térmico dos polímeros em condições de incêndio. Polímeros: Ciência e Tecnologia, 8(1), 23-38. http://dx.doi.org/10.1590/ S0104-14281998000100005.

6. Mouritz, A. P., \& Gibson, A. G. (2006). Fire properties of polymer composite materials. Ontario: Springer.

7. Martínez, D. R., \& Carbajal, G. G. (2010). Hidróxidos dobles laminares: arcillas sintéticas con aplicaciones en nanotecnología. Avances en Química, 7(1), 87-99.

8. Taviot-Gueho, C., Illaik, A., Vuillermoz, C., Commereuc, S., Verney, V., \& Leroux, F. (2007). LDH-dye hybrid material as coloured filler into polystyrene: structural characterization and rheological properties. Journal of Physics and Chemistry of Solids, 68(5-6), 1140-1146. http://dx.doi.org/10.1016/j. jpcs.2007.03.003.
9. Matusinovic, Z., Lu, H., \& Wilkie, C. A. (2012). The role of dispersion of LDH in fire retardancy: the effect of dispersion on fire retardant properties of polystyrene/Ca-Al layered double hydroxide nanocomposites. Polymer Degradation \& Stability, 97(9), 1563-1568. http://dx.doi.org/10.1016/j. polymdegradstab.2012.07.020.

10. Crepaldi, E. L., \& Valim, J. B. (1998). Hidróxidos duplos lamelares: síntese, estrutura, propiedades e aplicações. Quimica Nova, 21(3), 300-311. http://dx.doi.org/10.1590/ S0100-40421998000300011.

11. Zammarano, M., Franceschi, M., Bellayer, S., Gilman, J. W., \& Meriani, S. (2005). Preparation and flame resistance properties of revolutionary self-extinguishing epoxy nanocomposites based on layered double hydroxides. Polymer, 46(22), 93149328. http://dx.doi.org/10.1016/j.polymer.2005.07.050.

12. Félix, T. (2010). Produção de compósitos e nanocompósitos de poliestireno de alto impacto com propriedades antichamas (Dissertação de mestrado). Universidade Federal de Santa Catarina, Florianópolis.

13. Botan, R., Nogueira, T. R., \& Lona, L. M. F. (2011). Síntese e caracterização de nanocompósitos esfoliados de poliestireno: hidróxido duplo lamelar via polimerização in situ. Polímeros: Ciência e Tecnologia, 21(1), 34-38. http://dx.doi.org/10.1590/ S0104-14282011005000017.

14. Ding, P., \& Qu, B. (2005). Synthesis and characterization of exfoliated polystyrene/ZnAl layered double hydroxide nanocomposite via emulsion polymerization. Journal of Colloid and Interface Science, 291(1), 13-18. http://dx.doi. org/10.1016/j.jcis.2005.08.056. PMid:16165142.

15. Bujdosó, T., Hornok, V., \& Dékány, I. (2011). Thin films of layered double hydroxide and silver-doped polystyrene particles. Applied Clay Science, 51(3), 241-249. http://dx.doi. org/10.1016/j.clay.2010.11.026.

16. Zhao, C., Qin, H., Gong, F., Feng, M., Zhang, S., \& Yang, M. (2005). Mechanical, thermal and flammability properties of polyethylene/clay nanocomposites. Polymer Degradation \& Stability, 87(1), 183-189. http://dx.doi.org/10.1016/j. polymdegradstab.2004.08.005.

17. Qiu, L., Chen, W., \& Qu, B. (2005). Structural characterisation and thermal properties of exfoliated polystyrene/ZnAl layered double hydroxide nanocomposites prepared via solution intercalation. Polymer Degradation \& Stability, 87(3), 433-440. http://dx.doi.org/10.1016/j.polymdegradstab.2004.09.009.

18. Nyambo, C., Kandare, E., Wang, D., \& Wilkie, C. A. (2008). Flame-retarded polystyrene: Investigating chemical interactions between ammonium polyphosphate and $\mathrm{MgAl}$ layered double hydroxide. Polymer Degradation \& Stability, 93(9), 1656-1663. http://dx.doi.org/10.1016/j.polymdegradstab.2008.05.029.

19. Duarte de Farias, A. M., Fraga, M. A., Oliveira, R. B., \& Oliveira, M. G. (2010). Propriedades mecânicas e térmicas de nanocompósitos de polipropileno e hidróxidos duplos lamelares. In Anais $19^{\circ}$ Congresso Brasileiro de Engenharia e Ciência dos Materiais (pp. 8413-8420). Campos do Jordão: CBECiMat.

20. Leroux, F., Meddar, L., Mailhot, B., Morlat-Thérias, S., \& Gardette, J. (2005). Characterization and photooxidative behaviour of nanocomposites formed with polystyrene and LDHs organo-modified by monomer surfactant. Polymer, 46(11), 3571-3578. http://dx.doi.org/10.1016/j.polymer.2005.03.044.

21. Wang, L., Su, S., Chen, D., \& Wilkie, C. A. (2009). Variation of anions in layered double hydroxides: effects on dispersion and fire properties. Polymer Degradation \& Stability, 94(5), 770781. http://dx.doi.org/10.1016/j.polymdegradstab.2009.02.003.

Enviado: Out. 24, 2013

Revisado: Set. 18, 2014

Aceito: Jan. 12, 2015 